\title{
Duality between coordinates and wave functions on noncommutative space
}

\author{
Ion V. Vancea*
}

October 31, 2018

\author{
Departamento de Física e Matemática, \\ Faculdade de Filosofia, Ciêcias e Letras de Ribeirão Preto USP, \\ Av. Bandeirantes 3900, Ribeirão Preto 14040-901, SP, Brasil
}

\begin{abstract}
The relation between coordinates and the solutions of the stationary Schrödinger equation in the noncommutative algebra of functions on $R^{2 N}$ is discussed. We derive this relation for a certain class of wave functions for which the quantum prepotentials depend linearly on the coordinates similarly to the commutative case. Also, the differential equation satisfied by the prepotentials is given.
\end{abstract}

*ion@dfm.ffclrp.usp.br 
Recently, it has been noted in $[1,2]$ that by introducing a functional on the space of the solutions of the stationary Schrödinger equation (and also the Klein-Gordon equation) which connects two linearly independent solutions and is called a quantum prepotential, one can express a space-time coordinate as function of the corresponding solutions of the Schrödinger equation and the prepotential. This method works well for any differential equation of second order with constant coefficients and constant Wronskian and also for a wide class of partial differential equations of second order with constant coefficients. Tentatives to locally generalize this construction to gravity and to the Dirac equation have been made in $[3,4,5]$ but it has not been found the same simple and clear structure as in the case of the second order equations. An inspection of the relations involved in the duality between the coordinates and the wave-functions shows that the duality is actually a Legendre transformation and suggests the physics should not change if one passes from one side of the duality to the other side. In a more concrete fashion, it has been postulated that systems with an arbitrary potential, if subjected to an Equivalence Postulate applied to the Hamilton-Jacobi equation, manifest an invariance under the Legendre transformation between the coordinates and momenta $[6,7,8,9,10,11,12,13,14]$. (For earlier related works see $[15,16,17,18,19,20,21]$.) This shows that the duality between the coordinates and wave functions possibly could be related to more deeper properties of the physical systems. On the other hand, it allows us to express the coordinates of space-time in terms of quantum quantities, which represents a new approach to the structure of space-time at high energies. From this point of view, the parametrization of space-time in terms of real numbers is secondary since it comes out of relations between prepotentials and wave functions which are quantum and thus more fundamental. This interpretation raises a series of interesting questions concerning the symmetries of space-time, the structure of space-time at the planckian scale, the properties of the quantum prepotentials and their form in other representations, and so on. We are going to address some of this questions in future works.

The aim of the present letter is to derive the duality between coordinates and wave functions at the Planck scale in the framework of the noncommutative space-time. Our motivation relies on the observation that the structure of the space-time at higher energies should be different from the classical picture only at the Planck scale where the gravity has the same strength as the other fundamental interactions. However, the best understanding of the fundamental interactions at this scale is presently given by the string theory. In a more general setting, the string theory induces a noncommutative structure of the space-time. This is due to the presence of the Kalb-Ramond field from the closed string spectra. The noncommutative structure is tractable in the particular case where the Kalb-Ramond field is constant [22]. Since in the limit of infinite string tension the strings behave as particles we expect to recover the particle Quantum Mechanics at some stage in this limit. This justifies the study of Quantum Mechanics on noncommutative spaces and sets out the framework of this study.

The noncommutative structure that emerges from the string theory is given by the following product [22] between the smooth functions on the Euclidean $R^{2 N}$

$$
(f * g)(x)=\left.e^{\frac{i}{2} \theta^{m n} \partial_{m}^{(1)} \partial_{n}^{(2)}} f\left(x_{1}\right) g\left(x_{2}\right)\right|_{x_{1}=x_{2}=x} .
$$

Here, $\theta^{m n}=-\theta^{n m}$ are of order of $L^{2}$, where $L$ is the fundamental length of the problem. In what follows we take $L=l_{P}$, the Planck length, according to the above discussion. 
The relation (1) can be interpreted as a map between the algebra of commutative smooth functions $\mathcal{F}\left(R^{N}\right)$ on $R^{N}$ denoted by $\mathcal{A}_{\bullet}$ and some infinite dimensional noncommutative associative algebra $\mathcal{A}_{\star}$. The map (1) associates to each element $f \in \mathcal{A}_{\bullet}$ an element denoted by the same symbol in $\mathcal{A}_{\star}$ and called a noncommutative function on $R^{2 N}$ and allows us to interpret $\mathcal{A}_{\star}$ as a deformation of $\mathcal{A}_{\bullet}$ with the noncommutative parameters $\theta^{m n}$. The two interpretations of the relation (1), i. e. as a new product rule on the space of smooth functions and as a map between two algebras, are both useful to study different aspects of noncommutativity of the space-time.

Now let us define our general setting. The string theory describes the fundamental interactions at the Planck scale. Therefore, in this paper we consider the string length fixed to the Planck length. However, in infinite tension limit, the string length goes to zero. Thus, the Planck length also goes to zero, which is the limit of Quantum Mechanics. Consequently, the essential noncommutative contribution to the star product (1) comes from the linear terms in $\theta^{m n}$ 's. This fixes our approximation. In particular, one can easily check out that (1) implies

$$
\left[x^{m}, x^{n}\right]_{\star}=i \theta^{m n} \text {. }
$$

The above relation is valid not only in the linear approximation of the star product, but also in its general form. If we treat $x^{m}$ s as cordinates on the noncommutative $R^{2 N}$ manifold, their noncommutativity raises the question of the corresponding induced structure in the tangent and the cotangent spaces (see, for example [23, 24] for recent results on geometry with noncommutative parameters). More important for us, it posses the problem of choosing either noncommutative or commutative linear momenta associated with $x^{m}$ 's. As discussed in $[25,26,27,28,29,30]$, the noncommutative momenta lead to usual commutative systems in the presence of a magnetic field while a genuine noncommutative situation is obtained if one considers commuting momenta. This remark, together with the quantization procedure, justifies working with the following algebra

$$
\begin{aligned}
& {\left[x^{m}, p_{n}\right]_{\star}=i \hbar \delta_{n}^{m}} \\
& {\left[p_{m}, p_{n}\right]_{\star}=0}
\end{aligned}
$$

Together with the relation (1), the commutator (4) states that the linear momenta are independent of $x^{m}$ 's. The stationary Schrödinger equation is constructed by analogy with the commutative case and has the following form [31]

$$
H(x, p) \star \Psi(x)=E \Psi(x) .
$$

Here, the wave-function $\Psi$ belongs to the noncommutative algebra $\mathcal{A}_{\star}$. It is important to note that the equation (5) should be interpreted as a formal algebraic equation since the noncommutative coordinates do not form a complete set of observables. The analogy with the commutative case will be our guide in the search of a duality between the noncommutative coordinates (2) and the solutions of the equation (5) in the algebra $\mathcal{A}_{\star}$. This duality represents an extension of the similar result from the commutative case. Also, it is an interesting mathematical problem by itself.

Consider the explicit form of the Schrödinger equation (5)

$$
\left[-\frac{\hbar^{2}}{2 M} \sum_{m=1}^{2 N} \partial_{m}^{2}+V\right] \star \Psi=E \Psi
$$


where $V(x)$ is an arbitrary function from $\mathcal{A}_{\star}$ and $M$ is the mass of particle. As can be easily checked out, the star product in the kinetic term is equal to the commutative product. Following the commutative case [1], we treat the coordinate $x^{k}$ for $k=1,2, \ldots, 2 N$ as a variable and fix the coordinates $x^{s}$ for $s \neq k$. Thus, the equation (6) splits in to a system of $2 N$ dependent differential equations of second order in the algebra $\mathcal{A}_{\star}$. The coordinates $x^{s}$ play the role of paramenters in each of these equations. Since the inverse of any nonvanishing function is well defined in the noncommutative algebra $\mathcal{A}_{\star}$ being equal to its inverse in the commutative algebra, one can write the system from (6) under the following form

$$
\left(-\frac{\hbar^{2}}{2 M} \partial_{k}^{2}+V_{k} \star \Psi\right)(x)=E \Psi(x),
$$

and the potential in the $x^{k}$ direction has form

$$
V_{k}(x)=V(x)-\frac{\hbar^{2}}{2 M} \sum_{s \neq k}^{2 N}\left(\partial_{s}^{2} \Psi \star \Psi^{-1}\right)(x),
$$

for all $k$. In order to establish a duality between the coordinates and the wave functions, we need two linearly independent solutions of the equation (7). In the commutative case, the existence of them is guaranteed by theorems concerning the Wronskian function which are not always available in the noncommutative algebra. In fact, as on can see from (1), the Schrödinger equation contains derivatives of arbitrary large order. Even if we limit ourselves to the first order in $\theta$ 's, the corresponding equation is of second order with nonvanishing coefficient of the first order derivatives. Therefore, our problem is to find the conditions for which the solutions of the equation (7) admit a dual representation in terms of coordinates.

Let us assume that there are two solutions of the equation (7) denoted by $\Psi_{k}$ and $\tilde{\Psi}_{k}$. If they are linearly dependent, i. e. there are two nonzero complex numbers $c_{k}$ and $\tilde{c_{k}}$ such that the relations

$$
\Psi_{k}=-\frac{\tilde{c}_{k}}{c_{k}} \tilde{\Psi}_{k} \quad, \quad \partial_{k} \Psi_{k}=-\frac{\tilde{c}_{k}}{c_{k}} \partial_{k} \tilde{\Psi}_{k}
$$

hold simultaneously, then the following function

$$
W_{N C}^{k}=\partial_{k} \Psi_{k} \star \tilde{\Psi}_{k}-\partial_{k} \tilde{\Psi}_{k} \star \Psi_{k}
$$

is zero at each point $x \in R^{2 N}$. This suggest using the noncommutative wronskian as defined by the above relation as a criterion for the behaviour of the two solutions. Assume now that the two solutions of the equation (7) are linearly independent. The derivative of $W_{N C}^{k}$ with respect to the variable $x^{k}$ follows from the Leibniz' property of the derivative in $\mathcal{A}_{\star}$ and has the following form

$$
\partial_{k} W_{N C}^{k}(x)=\left[\partial_{k} \Psi_{k}, \partial_{k} \tilde{\Psi}_{k}\right]_{\star}(x)+\left\{\left(V_{k}-E\right) \star\left[\tilde{\Psi}_{k}, \Psi_{k}\right]_{\star}\right\}(x) .
$$

Here and in what follows we are taking $\hbar^{2} / 2 M=1$. Next, let us introduce the quantum prepotential defined as in the commutative case [1] by the following relation

$$
\tilde{\Psi}_{k} \equiv \frac{\partial \mathcal{F}^{k}\left[\Psi_{k}\right]}{\partial \Psi_{k}}
$$


The quantum prepotential $\mathcal{F}^{k}$ is a functional on the space of the solutions of the equation (7) for each $k$. In order to compute its derivative with respect to the coordinate $x^{k}$, one has to solve the ambiguity of ordering the terms in the chain product. Indeed, one has two nonequivalent possibilities for that, namely the left- and right-derivatives given by the following relations

$$
\partial_{k} \mathcal{F}_{L}^{k} \equiv \frac{\partial \mathcal{F}^{k}}{\partial_{k} \Psi_{k}} \star \partial_{k} \Psi_{k} \quad, \quad \partial_{k} \mathcal{F}_{R}^{k} \equiv \partial_{k} \Psi_{k} \star \frac{\partial \mathcal{F}^{k}}{\partial_{k} \Psi_{k}}
$$

It turns out that the symmetriezed derivative defined by

$$
\partial_{k}^{S} \mathcal{F}^{k}=\frac{1}{2}\left(\partial_{k} \mathcal{F}_{L}^{k}+\partial_{k} \mathcal{F}_{R}^{k}\right)
$$

satisfies the following relation

$$
2 \partial_{k}^{S} \mathcal{F}^{k}=\partial_{k}\left(\tilde{\Psi}_{k} \star \Psi_{k}\right)+W_{N C}^{k},
$$

which is similar to the one obtained in the commutative space [1]. Also, if one uses the symmetrized derivative (14) one can show that in the first order in $\theta$ 's

$$
\partial_{k}^{S} \mathcal{F}^{k}=\left.\partial_{k} \mathcal{F}^{k}\right|_{\mathcal{A}_{\bullet}}
$$

The above relation allows us to define the differential of the quantum prepotential as follows

$$
d \mathcal{F}^{k} \equiv \sum_{k=1}^{2 N} \partial_{k}^{S} \mathcal{F}^{k} d x^{k}
$$

It is straightforward to integrate out the relation (15). However, the result depends on the explicit form of the noncommutative wronskian which, in general, is not a constant function of $x^{k}$. Nevertheless, a similar result to the commutative case can be achieved by imposing that $W_{N C}^{k}$ satisfies

$$
\theta^{m n}\left\{\left(V_{k}-E\right) \partial_{m} \Psi_{k} \partial_{n} \tilde{\Psi}_{k}+\partial_{m} \partial_{k} \Psi_{k} \partial_{n} \partial_{k} \tilde{\Psi}_{k}\right\}=0
$$

The above relation represents a constraint to obtain a linear equation in $x^{k}$ after integration. If one has in the r.h.s. of the equation a different function on $x^{k}$, one has a different dependence of the coordinate $x^{k}$ on the corresponding wave function and prepotential. Just for completeness, let us give the expression of the noncommutative wronskian to the first order in $\theta$ 's

$$
W_{N C}^{k}(x)=W^{k}(x)+\frac{i}{2} \theta^{m n}\left[\frac{\partial^{2} \mathcal{F}^{k}}{\left(\partial \Psi_{k}\right)^{2}}(x)-\frac{\partial^{3} \mathcal{F}^{k}}{\left(\partial \Psi_{k}\right)^{3}}(x)\right] \partial_{m} \partial_{k} \Psi_{k}(x) \partial_{n} \Psi_{k}(x) .
$$

Here, $W^{k}$ represents the commutative wronskian. If the equation (18) is satisfied, the relation between the noncommutative coordinate $x^{k}$ and the wave function has the following form

$$
x^{k}=\mathcal{F}^{k}\left[\Psi_{k}\right]-\frac{1}{2} \tilde{\Psi}_{k} \star \Psi_{k}-f^{k}\left(x^{s}\right),
$$


where $f^{k}\left(x^{s}\right)$ is a function of the parameters $x^{s}, s \neq k$ only. The noncommutative algebra (2) and (3) imposes further constraints on the relation between the noncommutative potential and the wave function

$$
\begin{gathered}
{\left[\mathcal{F}^{k}\left[\Psi_{k}\right]-\frac{1}{2} \tilde{\Psi}_{k} \star \Psi_{k}-f^{k}\left(x^{s}\right), \mathcal{F}^{l}\left[\Psi_{l}\right]-\frac{1}{2} \tilde{\Psi}_{l} \star \Psi_{l}-f^{l}\left(x^{r}\right)\right]_{\star}=i \theta^{k l},} \\
{\left[\mathcal{F}^{k}\left[\Psi_{k}\right]-\frac{1}{2} \tilde{\Psi}_{k} \star \Psi_{k}-f^{k}\left(x^{s}\right), p_{l}\right]_{\star}=i \hbar \delta_{l}^{k} .}
\end{gathered}
$$

It is a simple exercise to write down the explicit form of these relations. They should be used together with the dynamical equation for $\mathcal{F}^{k}$ which can be obtained by replacing the derivative of the potential with respect to $\Psi$ in the Schrödinger equation for $\tilde{\Psi}_{k}$ [1]. Its explicit form is given by the following relation

$$
\begin{aligned}
& \operatorname{Symm}\left\{\frac{1}{2}\left(\mathcal{F}^{k(1)}-\mathcal{F}^{k(2)}\right)^{-1} \star\left[\operatorname{Symm}\left[\frac{1}{2}\left(\mathcal{F}^{k(1)}-\mathcal{F}^{k(2)}\right)^{-1} \star \mathcal{F}^{k(1)}\right]\right]\right\}(x) \\
& -\frac{2 M}{\hbar^{2}}\left\{\left(V_{k}-E\right) \star \mathcal{F}^{k(1)}\right\}(x)=0,
\end{aligned}
$$

where Symm indicates the symmetrized star product with respect to the two arguments. The upper index $(i)$ next to the quantum potential represents its derivative of the $i$ th order with respect to the wave function $\Psi_{k}$.

To conclude, we have derived the relation between the noncommutative coordinates in $R^{2 N}$ and the wave functions of the algebraic Schrödinger equation. If the noncommutative wronskian is constant, the relevant relations are (20), (21) and (22). The first of these relations expresses the coordinate - wave function duality. The second relation represents a set of constraints on the explicit form of the quantum prepotential in terms of solutions of the Schrödinger equation. These constraints should be enforced on the solution of the last equation which is the dual of the Schrödinger equation in the space of functionals $\mathcal{F}^{k}$. These results represent the generalization of the ones obtained in the commutative case in [1]. However, in the noncommutative case other possible relations are allowed. This can be seen from the equation (18) which enforces the linearity in $x^{k}$ in (20). By relaxing this condition, the dependence of $x^{k}$ on $\mathcal{F}^{k}$ is modified.

Acknowledgements I am grateful to M. A. Santos for useful discussions. This work was supported by the FAPESP Grant 02/05327-3.

\section{References}

[1] A. E. Faraggi and M. Matone, "A statistical interpretation of space and classicalquantum duality," Phys. Rev. Lett. 78, 163 (1997) [arXiv:hep-th/9606063].

[2] A. E. Faraggi and M. Matone, "Reply to comment on *Duality of $\mathrm{x}$ and psi in quantum mechanics*," arXiv:hep-th/9712245.

[3] I. V. Vancea, "Gravity And Duality Between Coordinates And Matter Fields," Phys. Lett. B 480, 331 (2000). 
[4] M. C. Abdalla, A. L. Gadelha and I. V. Vancea, "Duality between coordinates and Dirac field," Phys. Lett. B 484, 362 (2000) [arXiv:hep-th/0002217].

[5] M. A. De Andrade and I. V. Vancea, "Duality of coordinates and matter fields in curved spacetime," Phys. Lett. B 474, 46 (2000) [arXiv:gr-qc/9907059].

[6] G. Bertoldi, A. E. Faraggi and M. Matone, "Equivalence principle, higher dimensional Moebius group and the hidden antisymmetric tensor of quantum mechanics," Class. Quant. Grav. 17, 3965 (2000) [arXiv:hep-th/9909201].

[7] A. E. Faraggi and M. Matone, "The Equivalence Postulate of Quantum Mechanics," Int. J. Mod. Phys. A 15, 1869 (2000) [arXiv:hep-th/9809127].

[8] A. E. Faraggi and M. Matone, "Equivalence Principle: Tunnelling, Quantized Spectra and Trajectories from the Quantum HJ Equation," Phys. Lett. B 445, 357 (1999) [arXiv:hep-th/9809126].

[9] A. E. Faraggi and M. Matone, "Equivalence Principle, Planck Length and Quantum Hamilton-Jacobi Equation," Phys. Lett. B 445, 77 (1998) [arXiv:hep-th/9809125].

[10] A. E. Faraggi and M. Matone, "Quantum transformations," Phys. Lett. A 249, 180 (1998) [arXiv:hep-th/9801033].

[11] A. E. Faraggi and M. Matone, "The Equivalence Principle of Quantum Mechanics: Uniqueness Theorem," Phys. Lett. B 437, 369 (1998) [arXiv:hep-th/9711028].

[12] A. E. Faraggi and M. Matone, "Quantum mechanics from an equivalence principle," Phys. Lett. B 450, 34 (1999) [arXiv:hep-th/9705108].

[13] M. Matone, "Equivalence postulate and the quantum potential of two free particles," arXiv:hep-th/0212260.

[14] M. Matone, "Equivalence postulate and quantum origin of gravitation," Found. Phys. Lett. 15, 311 (2002) [arXiv:hep-th/0005274].

[15] E. R. Floyd, "Modified Potential And Bohm's Quantum Mechanical Potential," Phys. Rev. D 26 (1982) 1339.

[16] E. R. Floyd, "Arbitrary Initial Conditions Of Nonlocal Hidden Variables," Phys. Rev. D 29, 1842 (1984).

[17] E. R. Floyd, "Where and why the generalized Hamilton-Jacobi representation describes microstates of the Schrödinger wave function," Found. Phys. Lett. 9, 489 (1996) [arXiv:quant-ph/9707051].

[18] E. R. Floyd, "Reflection time and the Goos-Haenchen effect for reflection by a semi-infinite rectangular barrier," Found. Phys. Lett. 13 (2000) 235 [arXiv:quantph/9708007]. 
[19] R. W. Carroll, "Some remarks on quantum theory and integrable systems," arXiv:hepth/9607219.

[20] R. W. Carroll, "Further remarks on quantum mechanics and integrable systems," arXiv:hep-th/9610216.

[21] R. W. Carroll, "Two kinds of duality," arXiv:hep-th/9702138.

[22] N. Seiberg and E. Witten, "String theory and noncommutative geometry," JHEP 9909, 032 (1999) [arXiv:hep-th/9908142].

[23] C. K. Zachos, arXiv:hep-th/0008010.

[24] S. I. Vacaru, "Noncommutative Finsler geometry, gauge fields and gravity," arXiv:math$\mathrm{ph} / 0205023$.

[25] C. Sochichiu, "A note on noncommutative and false noncommutative spaces," arXiv:hep-th/0010149.

[26] C. Acatrinei, "Comments on Noncommutative Particle Dynamics," arXiv:hepth/0106141.

[27] R. P. Malik, "Dynamics in a noncommutative space," arXiv:hep-th/0302224.

[28] J. M. Romero, J. A. Santiago and J. D. Vergara, "Newton's second law in a noncommutative space," Phys. Lett. A 310, 9 (2003) [arXiv:hep-th/0211165].

[29] P. A. Horvathy, "The non-commutative Landau problem, and the Peierls substitution," Annals Phys. 299, 128 (2002) [arXiv:hep-th/0201007].

[30] R. Banerjee, "Dissipation and Noncommutativity in Planar Quantum Mechanics," Mod. Phys. Lett. A 17, 631 (2002) [arXiv:hep-th/0106280].

[31] L. Mezincescu, "Star Operation in Quantum Mechanics," arXiv:hep-th/0007046. 